\title{
Caregiver bereavement outcomes in advanced cancer: associations with quality of death and patient age
}

\author{
Kenneth Mah ${ }^{1} \cdot$ Nadia Swami $^{1}$ - Ashley Pope ${ }^{1} \cdot$ Craig C. Earle $^{2,3} \cdot$ Monika K. Krzyzanowska $^{3,4} \cdot$ Rinat Nissim $^{1,5}$. \\ Sarah Hales ${ }^{1,5} \cdot$ Gary Rodin $^{1,5,6} \cdot$ Breffni Hannon ${ }^{1,3,7}$. Camilla Zimmermann ${ }^{1,3,5,6,7}$ (])
}

Received: 26 April 2021 / Accepted: 31 August 2021 / Published online: 9 September 2021

(c) The Author(s), under exclusive licence to Springer-Verlag GmbH Germany, part of Springer Nature 2021

\begin{abstract}
Purpose We investigated relationships between domains of quality of dying and death in patients with advanced cancer and their caregivers' bereavement outcomes and the moderating effect of patient age at death.

Methods Bereaved caregivers of deceased patients with advanced cancer who had participated in an early palliative care trial completed measures of grief (Texas Revised Inventory of Grief [TRIG]), complicated grief (Prolonged Grief Inventory [PG-13]), and depression (Center for Epidemiologic Studies-Depression [CESD-10]). They also completed the Quality of Dying and Death measure (QODD), which assesses patients' symptom control, preparation for death, connectedness with loved ones, and sense of peace with death.

Results A total of 157 bereaved caregivers completed the study. When patient age $\times$ QODD subscale interactions were included, greater death preparation was related to less grief at patient death (past TRIG: $\beta=-.25, p=.04$ ), less current grief (present TRIG: $\beta=-.26, p=.03$ ), less complicated grief (PG-13: $\beta=-.37, p=.001$ ), and less depression (CESD-10: $\beta=-.35, p=.005$ ). Greater symptom control was related to less current grief (present TRIG: $\beta=-.27$, $p=.02$ ), less complicated grief (PG-13: $\beta=-.24, p=.03$ ), and less depression (CESD-10: $\beta=-.29, p=.01$ ). Significant patient age $\times$ connectedness interaction effects for current grief (present TRIG: $\beta=.30, p=.02$ ) and complicated grief (PG-13: $\beta=.29, p=.007$ ) indicated that, with less connectedness, younger patient age at death was associated with greater caregiver grief.

Conclusion Better end-of-life death preparation and symptom control for patients with cancer may attenuate later caregiver grief and depression. Less connectedness between younger patients and their families may adversely affect caregiver grief.
\end{abstract}

Keywords Age factors · Bereavement $\cdot$ Cancer $\cdot$ Caregivers $\cdot$ End-of-life care $\cdot$ Quality of dying and death

Camilla Zimmermann

camilla.zimmermann@uhn.ca

1 Department of Supportive Care, Princess Margaret Cancer Centre, University Health Network, Toronto, ON, Canada

2 Odette Cancer Centre, Sunnybrook Health Sciences Centre, Toronto, ON, Canada

3 Division of Medical Oncology, Department of Medicine, University of Toronto, Toronto, ON, Canada

4 Department of Medical Oncology and Hematology, Princess Margaret Cancer Centre, University Health Network, Toronto, ON, Canada
5 Department of Psychiatry, University of Toronto, Toronto, ON, Canada

6 Princess Margaret Research Institute, Princess Margaret Cancer Centre, University Health Network, Toronto, ON, Canada

7 Division of Palliative Medicine, Department of Medicine, University of Toronto, Toronto, ON, Canada 


\section{Introduction}

Family caregivers play a central role in the care of patients with advanced cancer throughout the course of illness, including at the end of life [1]. However, the stress of caring for severely ill loved ones, coupled with their subsequent death, can engender significant caregiving burden and long-term emotional distress after patient death, even when caregivers report satisfaction from providing care [2]. A recent meta-analysis reported that $17 \%$ of bereaved caregivers had symptoms of prolonged grief disorder [3]. Prolonged grief, also termed complicated grief, is defined as an intense grief reaction that is distinct from depression, that persists for at least 6 months after the death of the loved one, that can be characterized by difficulty accepting the loss and moving on with life and by traumatic distress symptoms such as numbness, and that is associated with impaired daily functioning [4].

Caregiver bereavement outcomes can vary depending on patient-related factors such as patient quality of dying and death at the end of life [5-8] and patient age at death [8, 9]. Patient quality of dying and death, a multidimensional, subjectively determined construct reflecting patients' dying experiences in the last days of life [10], may influence caregiver bereavement because family caregivers may be distressed by patients' suffering or feel responsible for supporting patients' quality of dying and death [11, 12]. Caregivers' perceptions of poor patient quality of dying and death and traumatic patient death have been linked to depression and complicated grief in bereaved caregivers [5-8, 11], whereas the sense of having done one's best for the patient has been associated with less regret and distress [12]. However, these studies investigated the impact of overall quality of dying and death [5, 6], general end-of-life distress [8], or individual items from quality of dying and death measures [7] without consideration for the conceptual domains of quality of dying and death the items might reflect. It remains unclear whether important, diverse conceptual domains of quality of dying and death, such as symptom control, practical preparations for death, relationships with loved ones, and a sense of peace with death as measured by the Quality of Dying and Death (QODD) questionnaire [13-15], differ in their impact on caregiver bereavement outcomes. Identifying the extent to which particular domains of quality of dying and death influence caregiver bereavement outcomes can inform clinical care and tailoring of interventions to attend to important domains.

Younger patient age has also been associated with greater caregiver depression [8] and grief or complicated grief $[9,16]$. The association between younger patient age and worse caregiver bereavement outcomes may reflect the developmentally unexpected nature of dying at a young age and the greater difficulty caregivers may consequently experience with accepting and coping with the loss [16, 17]. Younger patients and their families have reported greater difficulty facing disease progression and impending death [17], less death acceptance [18], and greater existential distress due to the perceived prematurity of their terminal illness $[17,19]$. It might be expected that patient quality of dying and death would affect caregiver bereavement more adversely with younger than older patient age at death, but whether patient age at death moderates the relationship between quality of dying and death and caregiver bereavement has not been examined.

The purpose of the present study was to investigate the associations between specific domains of patient quality of dying and death and caregiver bereavement outcomes in advanced cancer and to examine the moderating effect of patient age on these associations. We hypothesized that the associations between worse patient quality of dying and death and greater caregiver grief symptoms would be stronger when patients died at younger than older ages.

\section{Methods}

We combined data from our earlier cluster-randomized controlled trial of an early palliative care (EPC) intervention for patients diagnosed with advanced cancer [20] with data from a follow-up study of bereavement outcomes of these patients' caregivers after trial completion.

\section{Participants}

Participants from the EPC trial were patients diagnosed with advanced cancer and recruited from outpatient clinics at the Princess Margaret Cancer Centre, University Health Network (UHN), in Toronto, Canada, between December 2006 and February 2011. Inclusion criteria were age $\geq 18$ years, stage IV cancer (or stage III with poor prognosis), prognosis of 6-24 months' estimated survival, and oncologist-rated Eastern Cooperative Oncology Group measure of performance status (ECOG) [21] of 0-2. Patient exclusion criteria included indication of cognitive impairment (Short Orientation-Memory-Concentration Test score $<20$ or $>10$ errors) and insufficient English comprehension to participate.

After the trial, caregivers previously identified by trial participants or listed in their medical charts were approached at least 6 months after patient death about participating in the caregiver bereavement study. The bereavement study was conducted between October 2011 and May 2015. Caregiver exclusion criteria for both the trial and bereavement study included age $<18$ years and insufficient English comprehension. 


\section{Measures}

For the bereavement study, questionnaires were developed to collect caregiver sociodemographic characteristics and retrospective data about the caregiving role during the patients' last days of life. Patient age at death was recorded. Caregivers completed self-report questionnaires measuring the patients' quality of dying and death and their own grief and mood.

Caregivers completed a validated shortened version of the 33-item Quality of Dying and Death (QODD) questionnaire $[13,15]$ to report on patients' quality of dying experience within the last 7 days of life (or last month of life, if the patient remained unconscious or unresponsive during the last week). This version retained 17 of the original 31 content items, as well as a rating of overall quality of life at the end of life (last 7 days of life) [14]. It provides a total score and four subscale scores: symptom control, which assesses symptom management (pain under control, control over what was going on, able to breathe comfortably); preparation, which assesses practical preparations for death (e.g., visits with spiritual advisor, funeral arrangements in order, health care costs covered); connectedness, which assesses relationships with loved ones (sharing physical expressions of affection, spending time with family and friends); and transcendence, which assesses a sense of peace with death (unafraid of dying, at peace with dying, untroubled about strain on loved ones) [14]. Bereaved caregivers retrospectively rate the patients' experience of each item on a 0 (terrible experience) to 10 (almost perfect experience) scale; higher scores reflect better quality of dying and death. For the current study, prorated subscale scores, standardized to a 0-100 scale, were calculated for those who responded to at least $50 \%$ of each subscale's items. The QODD is the most widely used, best validated measure of quality of dying and death [22] and has demonstrated good psychometric characteristics with bereaved family members [13], including in palliative care settings [23]. The 17-item version showed good model fit of the four-factor structure in mixed-disease samples (mostly cancer diagnoses), and the subscales correlated significantly with ratings of overall quality of dying in the last week of life and overall quality of moment of death [14].

We assessed caregiver bereavement outcomes using three measures. The 21-item Texas Revised Inventory of Grief (TRIG) [24] scale was used to assess caregivers' past grief reactions at the time of patient death and present grief reactions. Bereaved individuals rate eight past-grief items and 13 present-grief items on how well each item applies to them, from 1 (completely true) to 5 (completely false); to facilitate interpretation of results, items were reverse-scored so that higher scores indicate greater grief. Past and present total summed scores were calculated. The scale has demonstrated good internal consistency, construct validity [25], and relationships with pre-death grief [26] for bereaved caregivers in palliative care settings.

The 13-item Prolonged Grief Inventory (PG-13) [27], which comprises diagnostically informative items from the validated Inventory of Complicated Grief [28], was used to assess the extent of prolonged grief. The first five questions ask caregivers to rate frequency of grief reactions within the past month from 1 (not at all) to 5 (several times a day). The next eight questions ask caregivers to rate current negative reactions and difficulties with moving on from 1 (not at all) to 5 (overwhelming). A total summed score was calculated using items 4-12, which describe symptoms associated with prolonged grief disorder [27]; higher scores indicate greater grief. The PG-13 has demonstrated good psychometric properties with bereaved family members, including support for a single-factor structure $[29,30]$ and evidence for prolonged grief disorder as distinct from depression [31].

To assess depressive symptoms, we used the 10 -item version [32] of the 20-item Center for Epidemiologic StudiesDepression Scale (CESD) [33]. This shortened version, the CESD-10, was derived and validated using data from healthy older adults [32]. Items are rated on frequency of occurrence during the past week from 0 (rarely or none of the time) to 3 (most or all of the time), with higher scores indicating worse depressive symptoms. The CESD and CESD-10 are often used to assess bereavement in caregivers of patients with advanced cancer [31, 34, 35].

\section{Procedure}

The UHN Research Ethics Board approved the EPC trial protocol [20], which was registered with ClinicalTrials.gov (\#NCT01248624), and the follow-up bereaved caregiver study (REB \#06-0525-CE). In the trial, patients were cluster-randomized to receive either the EPC intervention or usual cancer care. Outcomes from the EPC trial have been reported previously $[20,36]$. Patients provided written informed consent for the trial.

At least 6 months after patient death, eligible caregivers were informed by mail about the bereaved caregiver study. Those who did not opt out were contacted to obtain written informed consent. They were mailed the study questionnaire package to complete either on their own and return by mail or to complete by telephone with research staff. A $\$ 5$ coffee gift card was provided to compensate respondents for their participation.

\section{Statistical analyses}

Trial baseline data [20] for those patients linked with bereaved caregiver study participants were combined with 
the caregiver data for analyses, which were conducted using SPSS version 25. Alpha was set to 0.05 .

Univariate analyses (Pearson's correlations, ANOVAs, chi-square tests) were conducted to identify significant sociodemographic and medical correlates of the main caregiver bereavement outcomes (Past TRIG, Present TRIG, PG-13, and CESD-10). Significant correlates $(p<0.05)$ were included as covariates in the main analyses of their respective bereavement outcomes. Time since patient death and EPC trial treatment group were additionally included as covariates in all main analyses.

Separate hierarchical multiple regression analyses were conducted with each bereavement outcome to investigate the relationships between the QODD subscales and each bereavement outcome and the moderating effect of patient age at death. Patient age and QODD subscales were centered on their respective means to minimize collinearity between main and interaction effects. Correlates were entered sequentially in the following order: (1) identified covariates, (2) main effects of QODD subscales and patient age, and (3) two-way interaction effects of each QODD subscale with patient age. Predicted adjusted outcomes were saved to facilitate examination of significant interaction effects. Any significant interaction effect was plotted to compare the directions of the relationship between the QODD subscale and grief outcome involved across three patient age groups (youngest, intermediate, and oldest), which were created by dividing participants into tertiles based on the centered patient ages at death.

\section{Results}

\section{Participant characteristics}

Of 431 bereaved caregivers contacted (214 caregivers of usual care participants, 217 caregivers of EPC participants), 182 (42.2\%; 86 caregivers of usual care participants, 96 caregivers of EPC participants) consented to the bereaved caregiver study. Of the 182 consented caregivers, 157 (86.3\%; 75 caregivers of usual care participants, 82 caregivers of EPC participants) completed the study. Table 1 summarizes the characteristics of the 157 caregiver participants and the corresponding EPC trial patient participants. On average, caregivers and patients were just over 60 years of age (ranges: caregivers $=29-88$ years; patients $=28-88$ years), and patients were just under 64 years of age at death (range $=29-90$ years). The majority of caregivers had been spouses/partners of the deceased patients. The mean time since patient death was about 3 years, with a range of 0.64 to 4.89 years.

\section{Patient quality of dying and death and caregiver outcomes}

Patient quality of dying and death and bereaved caregiver outcomes are summarized in Table 2. Mean scores for the QODD subscales were better for preparation and connectedness than for symptom control and transcendence. Caregiver grief, as measured by the past and present TRIG, was moderate, whereas the PG-13 and the CESD-10 suggested lower levels of distress. There were no significant treatment-group differences in any of these outcomes.

\section{Impact of patient quality of dying and death on bereaved caregiver outcomes and moderating effect of patient age}

Table 3 summarizes the results of the regression analyses investigating the relationships between the four QODD subscales and bereavement outcomes.

\section{Past TRIG}

Entry of the main effects accounted for a small but significant proportion of variance in past TRIG scores $\left(\mathrm{R}^{2}=0.20\right.$, $p<0.001)$. Patients' age at death was not significant. The QODD preparation subscale was a significant correlate of past TRIG, with greater preparation being related to less grief at patient death $(\beta=-0.30, p=0.01)$. This relationship remained significant upon entry of the patient age $\times$ QODD subscale interactions $(\beta=-0.25, p=0.04)$, which accounted for a small, non-significant proportion of variance $\left(\mathrm{R}^{2}=0.03, p=0.40\right)$. None of the interaction effects was significant.

\section{Present TRIG}

Entry of the main effects accounted for a small but significant proportion of variance in present TRIG scores $\left(\mathrm{R}^{2}=0.17, p<0.001\right)$. Patients' age at death was a significant correlate, with older patient age being linked to less present grief $(\beta=-0.18, p=0.048)$. Two QODD subscales, symptom control $(\beta=-0.26, p=0.02)$ and preparation $(\beta=-0.26, p=0.03)$, were significantly associated with present TRIG, indicating that greater symptom control and preparation were both linked with less present grief.

Entry of the patient age $\times$ QODD subscale interaction effects accounted for a small, non-significant proportion of variance in present TRIG scores $\left(\mathrm{R}^{2}=0.04, p=0.20\right)$. Both symptom control $(\beta=-0.27, p=0.02)$ and preparation $(\beta=-0.26, p=0.03)$ remained significant in the same directions, but not patient age $(p=0.09)$. There was a significant patient age $\times$ connectedness interaction effect ( $\beta=0.30, p=0.02)$. Figure 1 illustrates the directions of 
Table 1 Baseline caregiver and patient characteristics

\begin{tabular}{|c|c|c|c|c|}
\hline \multirow[b]{2}{*}{ Characteristics } & \multicolumn{2}{|l|}{ Caregivers } & \multicolumn{2}{|l|}{ Patients } \\
\hline & $\begin{array}{l}\text { EPC } \\
(n=82)\end{array}$ & Usual care $(n=75)$ & $\begin{array}{l}\text { EPC } \\
(n=82)\end{array}$ & Usual care $(n=75)$ \\
\hline Age, mean years (SD) [range] & $\begin{array}{l}61.17(12.61) \\
{[30-88]}\end{array}$ & $\begin{array}{l}58.05(13.60) \\
{[29-87]}\end{array}$ & $\begin{array}{l}62.77(12.26) \\
{[28-88]}\end{array}$ & $\begin{array}{l}61.52(11.90) \\
{[35-88]}\end{array}$ \\
\hline Patient age at death & - & - & $\begin{array}{l}63.98(12.23) \\
{[29-90]}\end{array}$ & $\begin{array}{l}63.25(11.82) \\
{[36-89]}\end{array}$ \\
\hline \multicolumn{5}{|l|}{ Time since patient death } \\
\hline Mean years (SD) & - & - & $3.19(0.82)$ & $3.00(0.86)$ \\
\hline Median years (range) & - & - & $3.15(1.10-4.89)$ & $3.09(0.64-4.63)$ \\
\hline Female gender, $n(\%)$ & $58(70.7)$ & $51(68.0)$ & $43(52.4)$ & $41(54.7)$ \\
\hline \multicolumn{5}{|l|}{ Education, $n(\%)$} \\
\hline$\leq$ High school & $18(22.0)$ & $18(24.0)$ & $25(30.5)$ & $26(34.7)$ \\
\hline College/university/other & $64(78.0)$ & $57(76.0)$ & $56(68.3)$ & $48(64.0)$ \\
\hline (Missing) & $0(0.0)$ & $0(0.0)$ & $1(1.2)$ & $1(1.3)$ \\
\hline \multicolumn{5}{|l|}{ Employment status, $n(\%)$} \\
\hline Employed & $35(42.7)$ & $36(48.0)$ & $16(19.5)$ & $16(21.3)$ \\
\hline Unemployed & $4(4.9)$ & $3(4.0)$ & $11(13.4)$ & $7(9.3)$ \\
\hline Retired & $40(48.8)$ & $33(44.0)$ & $39(47.6)$ & $37(49.3)$ \\
\hline Disabled & $3(3.7)$ & $2(2.7)$ & $16(19.5)$ & $15(20.0)$ \\
\hline Student & $0(0.0)$ & $1(1.3)$ & $0(0.0)$ & $0(0.0)$ \\
\hline \multicolumn{5}{|l|}{ Annual income, $n(\%)$} \\
\hline$\leq \$ 14,999$ & $1(1.2)$ & $1(1.3)$ & $5(6.1)$ & $2(2.7)$ \\
\hline$\$ 15,000-29,999$ & $5(6.1)$ & $6(8.0)$ & $5(6.1)$ & $8(10.7)$ \\
\hline$\$ 30,000-59,999$ & $17(20.7)$ & $26(34.7)$ & $11(13.4)$ & $15(20.0)$ \\
\hline$\geq \$ 60,000$ & $53(64.7)$ & $37(49.3)$ & $28(34.1)$ & $30(40.0)$ \\
\hline (Missing) & $6(7.3)$ & $5(6.7)$ & $33(40.2)$ & $20(26.7)$ \\
\hline \multicolumn{5}{|l|}{ Marital status, $n(\%)$} \\
\hline Married/common-law & $27(32.9)$ & $22(29.3)$ & $61(74.4)$ & $58(77.3)$ \\
\hline Separated/divorced & $3(3.7)$ & $4(5.3)$ & $6(7.3)$ & $10(13.3)$ \\
\hline Widowed & $49(59.8)$ & $45(60.0)$ & $9(11.0)$ & $3(4.0)$ \\
\hline Single & $3(3.7)$ & $4(5.3)$ & $6(7.3)$ & $4(5.3)$ \\
\hline \multicolumn{5}{|l|}{ Relationship to patient, $n(\%)$} \\
\hline Spouse/partner & $51(62.2)$ & $47(62.7)$ & - & - \\
\hline Child & $16(19.5)$ & $18(24.0)$ & - & - \\
\hline Other & $15(18.3)$ & $10(13.3)$ & - & - \\
\hline \multicolumn{5}{|l|}{ Cancer site, $n(\%)$} \\
\hline Breast & - & - & $8(9.8)$ & $11(14.7)$ \\
\hline Gastrointestinal & - & - & $30(36.6)$ & $23(30.7)$ \\
\hline Genitourinary & - & - & $12(14.6)$ & $13(17.3)$ \\
\hline Gynecologic & - & - & $9(11.0)$ & $15(20.0)$ \\
\hline Lung & - & - & $23(28.0)$ & $13(17.3)$ \\
\hline \multicolumn{5}{|l|}{ Performance status (ECOG), $n(\%)$} \\
\hline 0 & - & - & $19(23.2)$ & $24(32.0)$ \\
\hline 1 & - & - & $57(69.5)$ & $49(65.3)$ \\
\hline 2 & - & - & $6(7.3)$ & $2(2.7)$ \\
\hline \multicolumn{5}{|l|}{ Place of death } \\
\hline Home & - & - & $32(39.0)$ & $22(29.3)$ \\
\hline Hospital & - & - & $9(11.0)$ & $15(20.0)$ \\
\hline PCU/hospice & - & - & $37(45.1)$ & $38(50.7)$ \\
\hline Long-term care home & - & - & $2(2.4)$ & $0(0.0)$ \\
\hline (Missing) & - & - & $2(2.4)$ & $0(0.0)$ \\
\hline
\end{tabular}

Notes. "EPC" and "usual care" refer to the treatment arms to which patient participants of the EPC clinical trial had been assigned; for caregivers, these terms thus refer to the treatment arms that had been assigned to the patients they cared for. EPC, early palliative care intervention group. ECOG, Eastern Cooperative Oncology Group performance status scale. $P C U$, inpatient palliative care unit. SD, standard deviation 
Table 2 Comparison of the QODD and caregiver bereavement outcomes reported by caregivers of patient trial participants in the early palliative care intervention and usual care trial arms

\begin{tabular}{|c|c|c|c|}
\hline Characteristics & $\begin{array}{l}\mathrm{EPC} \\
(n=82)\end{array}$ & Usual care $(n=75)$ & $p$ \\
\hline \multicolumn{4}{|c|}{ QODD subscales, mean scores (SD) [range $=0-100$ ] } \\
\hline Symptom control & $56.54(25.28)$ & $54.98(23.84)$ & .70 \\
\hline Preparation & $73.45(23.73)$ & $69.22(19.03)$ & .26 \\
\hline Connectedness & $76.75(24.17)$ & $75.14(22.02)$ & .67 \\
\hline Transcendence & $54.87(30.86)$ & $47.78(26.58)$ & .15 \\
\hline \multicolumn{4}{|c|}{ Caregiver bereavement outcomes, mean scores (SD) } \\
\hline Past TRIG [range $=0-40$ ] & $20.15(8.29)$ & $20.46(6.97)$ & .81 \\
\hline $\begin{array}{l}\text { Present TRIG } \\
{[\text { range }=0-65]}\end{array}$ & $41.82(11.06)$ & $42.49(9.87)$ & .69 \\
\hline PG-13 [range $=9-45]$ & $16.42(7.71)$ & $16.01(6.81)$ & .72 \\
\hline CESD-10 [range $=0-30]$ & $8.20(7.34)$ & $7.56(5.75)$ & .54 \\
\hline
\end{tabular}

Notes. "EPC" and "usual care" refer to the treatment arms that had been assigned to the patients the caregivers cared for. For the QODD and each caregiver bereavement outcome, ranges of possible scale scores are included in brackets. $E P C$, early palliative care intervention group. ECOG, Eastern Cooperative Oncology Group performance status scale. $P C U$, inpatient palliative care unit. $Q O D D$, Quality of Dying and Death questionnaire. Past TRIG, past grief (at patient death), Texas Revised Inventory of Grief. Present TRIG, present grief, Texas Revised Inventory of Grief. $P G-13$, Prolonged Grief Inventory. CESD-10, Center for Epidemiologic Studies-Depression Scale, 10 -item version. $S D$, standard deviation

the relationships between connectedness and present grief for patients who were of younger, intermediate, and older ages at death. With less connectedness at the end of life, present caregiver grief was the greatest with the youngest patient age group and the least with the oldest age group. With increasing connectedness, however, all three age groups converged to more intermediate levels of present caregiver grief.

\section{PG-13}

Entry of the main effects accounted for a small but significant proportion of variance in PG-13 scores $\left(\mathrm{R}^{2}=0.27\right.$, $p<0.001)$. Patients' age at death did not quite meet significance $(p=0.054)$, but the symptom control $(\beta=-0.23$, $p=0.03)$ and preparation $(\beta=-0.37, p=0.001)$ subscales were both significantly associated with less grief.

The interaction effects accounted for a small but significant amount of variance $\left(\mathrm{R}^{2}=0.06, p=0.03\right)$. Along with the significant symptom control $(\beta=-0.24, p=0.03)$ and preparation ( $\beta=-0.37, p=0.001)$ main effects, a significant patient age $\times$ connectedness interaction effect was observed $(\beta=0.29, p=0.007)$. Figure 2 , which illustrates the directions of this interaction effect for younger, intermediate, and older patient ages at death, displays a similar pattern to the corresponding interaction effect with present TRIG.

\section{CESD-10}

Entry of the main effects accounted for a small but significant amount of variance in CESD-10 scores $\left(\mathrm{R}^{2}=0.20\right.$, $p<0.001)$. Better symptom control $(\beta=-0.29, p=0.009)$ and preparation $(\beta=-0.36, p=0.002)$ were both significantly related to less depressive symptoms in caregivers. Entry of the interaction effects did not account for a significant amount of variance $\left(\mathrm{R}^{2}=0.01, p=0.87\right)$, and while both main effects of symptom control $(\beta=-0.29, p=0.01)$ and preparation $(\beta=-0.35, p=0.005)$ remained, none of the interaction effects was significant.

\section{Discussion}

In this study of bereavement outcomes of caregivers of patients with advanced cancer, greater preparation for death and better symptom control, as aspects of patient quality of dying and death, were linked to less caregiver grief and depression. In addition, less connectedness between patients and families near the end of life was associated with greater prolonged caregiver grief when patients were younger at death than when they were older, whereas greater connectedness was associated with less grief regardless of age.

Better patient preparation for death was consistently associated with less caregiver distress across all measures. The QODD preparation subscale encompasses practical factors such as funeral arrangements and meetings with religiousspiritual advisors. Although the QODD rates caregivers' perceptions of patients' preparation for death, caregivers' participation in such preparations might have contributed to their own preparedness for death and thus reduced bereavement morbidity [35]. Additionally, preparation for death may reflect an aspect of death acceptance in both patients and caregivers [37], which, in turn, may lessen complicated grief in bereaved caregivers [9]. The quality of patient-clinician communication may have an impact on preparedness [38], underlining the importance of ensuring that clinicians are adequately trained to have end-of-life conversations.

Symptom control at the end of life was also associated with less current grief and depression. Management of pain and other symptoms is a cornerstone of palliative care's mandate of relieving suffering and improving quality of life for both patients and their families [39]. In patients with advanced cancer, symptoms and quality of life deteriorate with disease progression and may exhibit steep declines in the last months of life [40]. Given the sense of responsibility caregivers often feel to ensure patients' comfort at the end of life [11,12], effective symptom management especially at the end of life may thus play a critical role in caregivers' later post-death adjustment. 


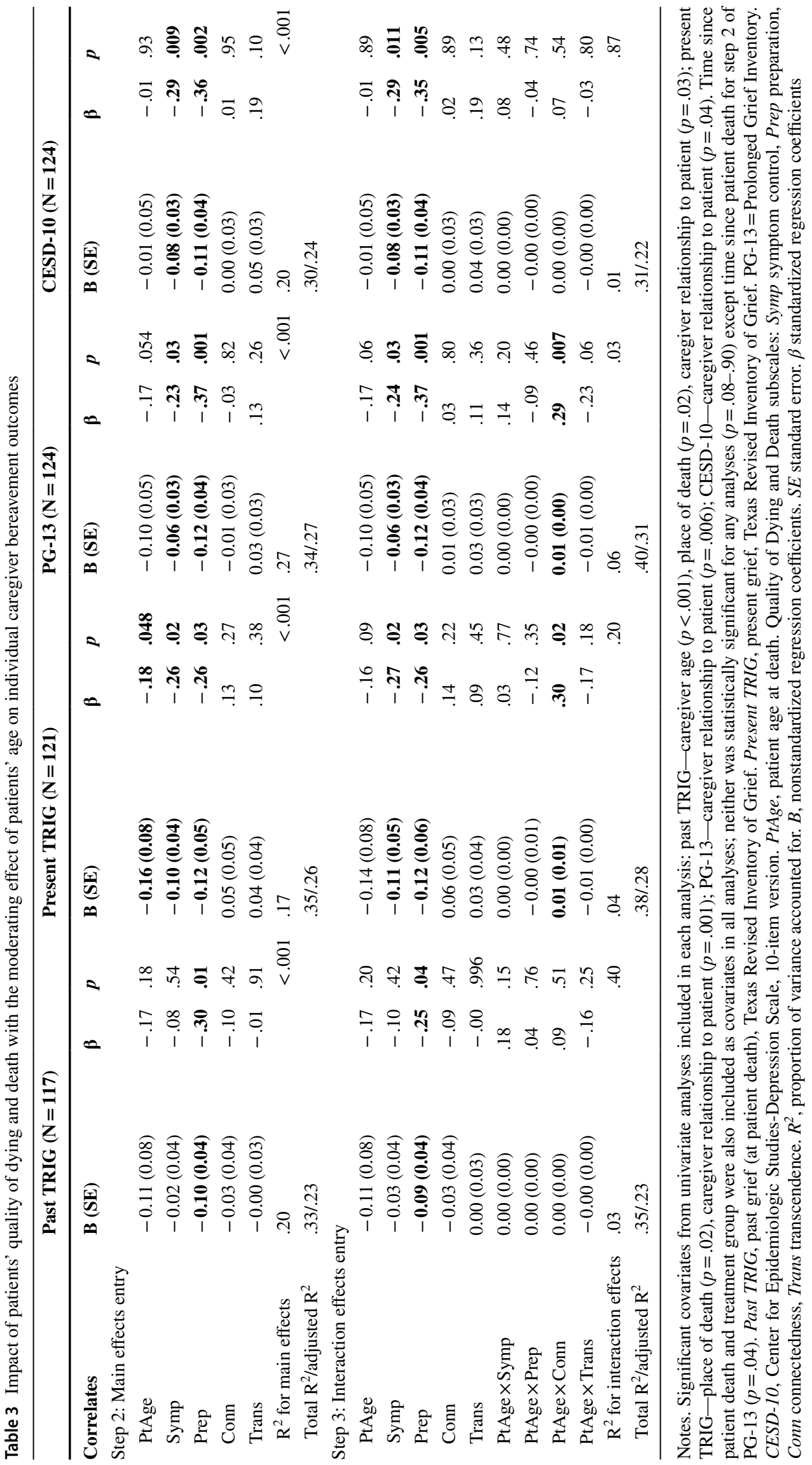




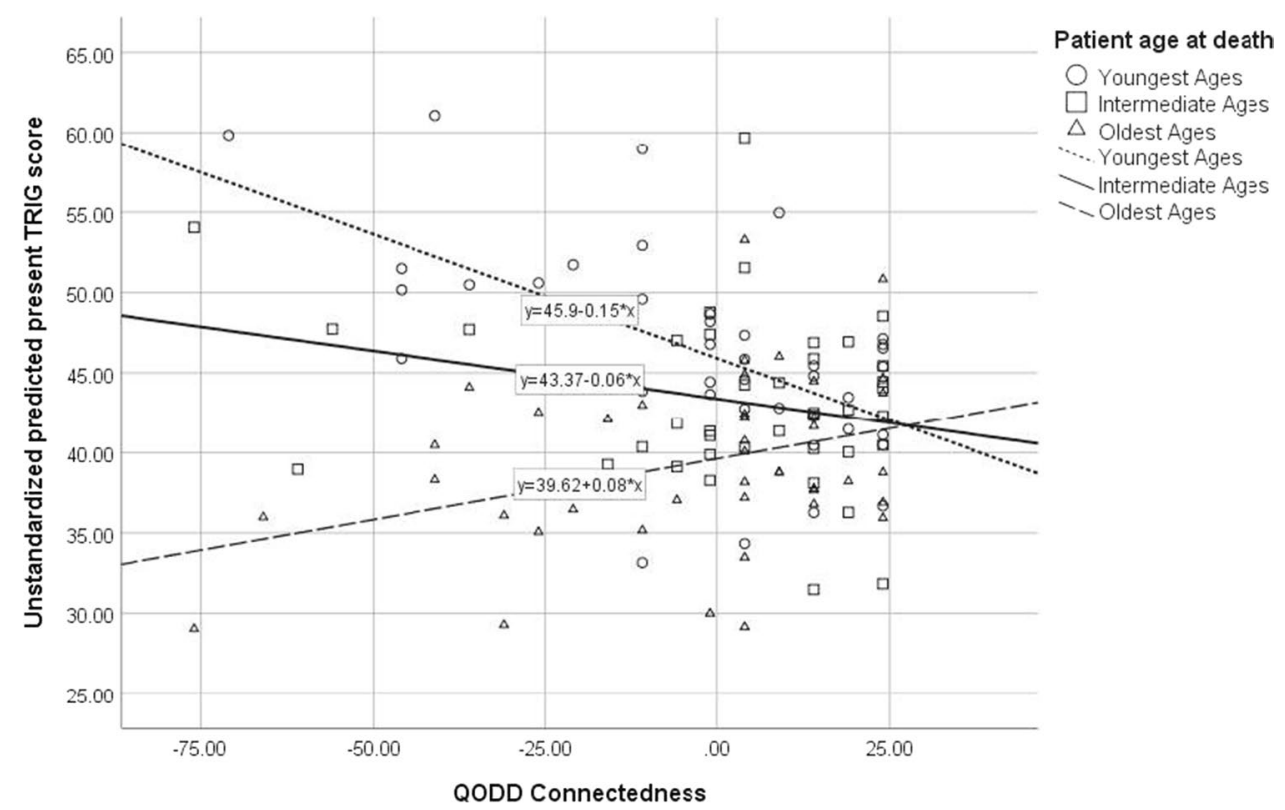

Fig. 1 Patient age at death $\times$ QODD Connectedness interaction effect on present TRIG. With less connectedness at the end of life, present caregiver grief was highest with the youngest patient age group and lowest with the oldest patient age group. With increasing connectedness, all three age groups were associated with more intermediate levels of present caregiver grief. QODD connectedness scores are centered scores. The present TRIG scores are predicted scores from the corresponding regression analyses. Groups representing youngest, intermediate, and oldest patient age at death are tertile groups. QODD, Quality of Dying and Death scale. Present TRIG, present grief, Texas Revised Inventory of Grief
Fig. 2 Patient age at death $x$ QODD Connectedness interaction effect on PG-13. With less connectedness at the end of life, caregiver grief was highest with the youngest patient age group and lowest with the oldest patient age group. With increasing connectedness, all three age groups were associated with lower levels of caregiver grief. QODD connectedness scores are centered scores. The PG-13 scores are predicted scores from the corresponding regression analyses. Groups representing youngest, intermediate, and oldest patient age at death are tertile groups. QODD, Quality of Dying and Death scale. PG-13, Prolonged Grief Inventory

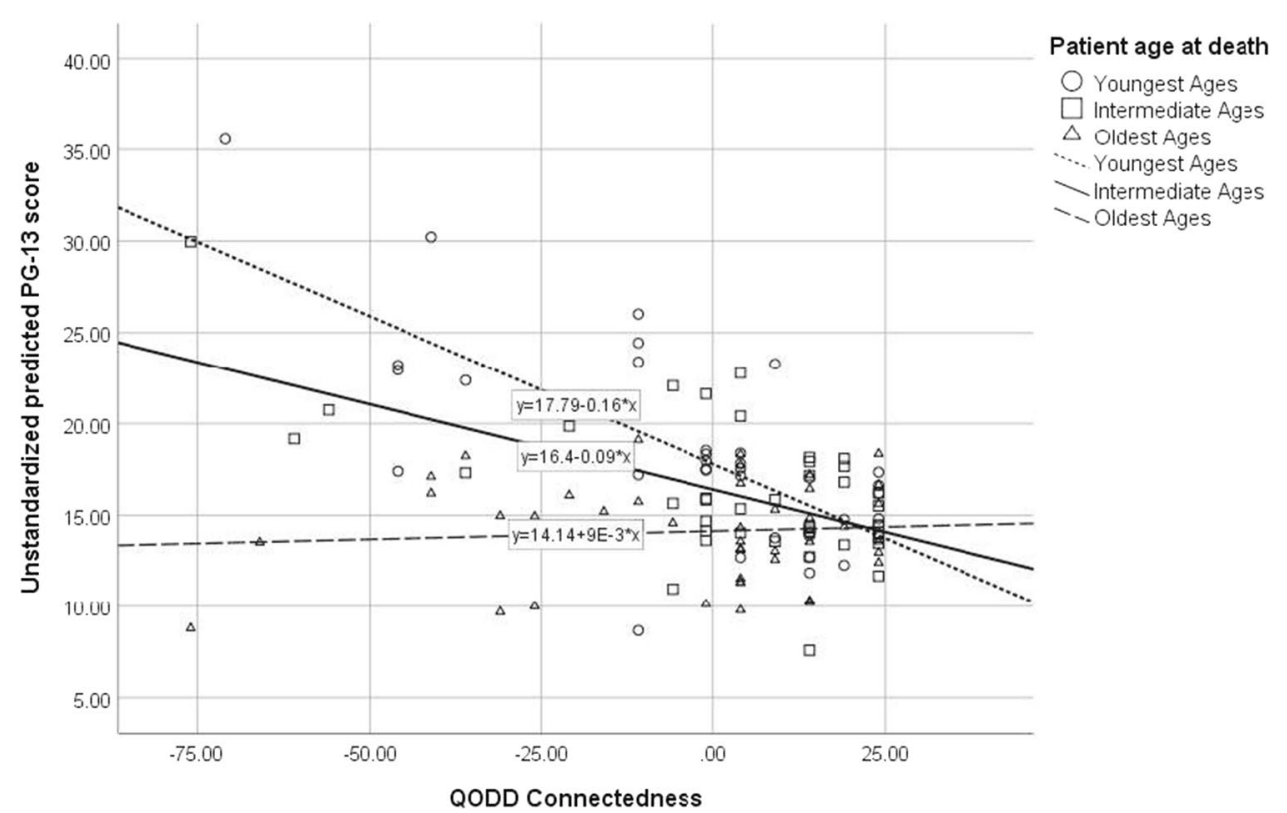

The relationship between interpersonal connectedness near the end of life and caregiver bereavement differed with patient age at death: less connectedness had a greater adverse impact on caregiver grief when patients died at younger than older ages, while greater connectedness was associated with less grief across ages. This relationship was evident only with the two measures of prolonged grief, suggesting the unique contribution of connectedness to these clinically relevant outcomes. Connectedness is a key factor in psychological and spiritual well-being in advanced cancer [41], with patients desiring the presence of family and family support near the end of life [41-43] and caregivers indicating the importance of spending time together and saying good-bye [44]. At the same time, dying individuals 
may withdraw from social relationships, and loved ones may similarly withdraw because of distress over witnessing their loved ones' deterioration or anticipatory grief [42]. Such issues could be exacerbated in younger patients and their families, as they confront the interruption of developmental and psychosocial roles and attainments or the loss of anticipated ones. Future studies should distinguish the specific issues that underlie the interacting effects of patient age and connectedness on caregiver bereavement, as these may become targets of psychotherapeutic intervention.

Our findings suggest that the emotional well-being of bereaved caregivers may be improved by adequate symptom management and supports for patients' preparations for the end of life, both of which are recognized elements of palliative care. Based on our findings, involving caregivers in end-of-life care may help to bolster their sense of having provided effective caregiving to their loved ones and to facilitate their preparation for their loved ones' death. Facilitating social interactions with loved ones may also be particularly beneficial in increasing younger patients' preparation for death [45]. The current COVID-19 pandemic and its distancing-related restrictions, in hampering caregivers from being with patients during provision of end-of-life care, may impede these aspects of quality of dying and death [46], however, and their impact on caregiver bereavement remains to be determined. Psychotherapeutic supports offered to patients and their families should address not only practical and psychological preparations for death but also the developmental and psychosocial challenges specific to younger patients.

Our study had limitations. Patients' quality of dying and death was rated by caregivers due to patients' inability to complete measures in the last days of life, but such proxy reporting may be affected by caregivers' current bereavement-related distress, as well as memory and recall bias [22], especially given mean caregiver recruitment time post-patient death of 3 years. Due to the variation in time since patient death, we included this variable in the main analyses, but no statistically significant impact on outcomes was demonstrated across entry of covariates, main effects, and interaction effects, except for PG-13 after entry of main effects $(p=0.04)$. All caregivers of trial patients were approached about the study after patient death, but, as only a subset participated, reduced power and generalizability are concerns. Although consistent, effects were small and did not explain beyond $40 \%$ of the variance in caregiver bereavement outcomes; using the full QODD may have increased power but would have increased burden. While we assessed the important effect of patient age, further studies should explore other potentially important moderators or mediators, including place of death or availability of support resources tailored to the unique needs of particular populations. Lastly, recruitment for the EPC trial and caregiver follow-up studies was completed approximately 10 and 6 years ago, respectively, and there have been changes in cancer treatment over time, such as more frequent use of immunotherapies. It would be interesting to compare the current results with a contemporary analysis to assess whether patient quality of dying and death and, in turn, caregiver bereavement have improved. However, we believe that the findings of the current study are still relevant, as the fundamental associations demonstrated between patient quality of dying and death and subsequent caregiver bereavement outcomes should not change substantially over time.

\section{Conclusion}

Caregiving can have a lasting emotional toll for caregivers long after patient death. Our findings highlight the contribution of patient quality of dying and death to caregiver bereavement and the particular concerns related to caregivers of younger patients. Supporting the quality of patient dying and death in advanced cancer should include providing ongoing symptom management, fostering mutuality in practical and psycho-spiritual preparations for death, and strengthening connectedness, particularly in younger patients and their families. Ensuring such high-quality end-of-life care may not only provide benefits at the end of life but also contribute to caregiver adjustment during bereavement.

Acknowledgements We thank the oncologists who referred patients to the trial and the clinical, research, and administrative staff of the Department of Supportive Care, Princess Margaret Cancer Centre, for their assistance with preparation of study materials, recruitment, and data entry and verification. We also extend our gratitude to the study participants, who gave their time despite the challenges they faced.

Author contribution All authors contributed to conception and design. AP and NS contributed to acquisition of data. KM completed data analysis and interpreted the data with input from CZ and LWL. KM and $\mathrm{CZ}$ drafted the article and all authors reviewed it critically for important intellectual content. All authors approved the final version and have participated sufficiently in the work to take responsibility for appropriate portions of the content.

Funding The study was supported by the Canadian Cancer Society [grant \#017257, \#020509, \#700862; CZ], the Canadian Institutes of Health Research [grant \#152996; CZ], and the Ontario Ministry of Health and Long-Term Care. CZ is also supported by the Rose Family Chair in Palliative Medicine and Supportive Care, Faculty of Medicine, University of Toronto. The funders of the original trial played no role in the study design, data collection and analysis, data interpretation, or writing of this report.

Data availability The corresponding author may be contacted regarding access to anonymized data. 
Code availability All statistical analyses were conducted with SPSS version 25 . The corresponding author may be contacted regarding access to codes for the conducted analyses.

\section{Declarations}

Ethics approval This study was performed in line with the principles of the Declaration of Helsinki. Approval was granted by the University Health Network Research Ethics Board (ethics approval number 06-0525-CE).

Consent to participate Informed consent was obtained from all individual participants included in the study.

\section{Consent for publication N/A.}

Competing interests The authors declare no competing interests.

\section{References}

1. Alam S, Hannon B, Zimmermann C (2020) Palliative care for family caregivers. J Clin Oncol 38:926-936. https://doi.org/10. 1200/jco.19.00018

2. Fasse L, Sultan S, Flahault C, MacKinnon CJ, Dolbeault S, Brédart A (2014) How do researchers conceive of spousal grief after cancer? A systematic review of models used by researchers to study spousal grief in the cancer context. Psychooncology 23:131-142. https://doi.org/10.1002/pon.3412

3. Komischke-Konnerup KB, Zachariae R, Johannsen M, Nielsen LD, O'Connor M (2021) Co-occurrence of prolonged grief symptoms and symptoms of depression, anxiety, and posttraumatic stress in bereaved adults: a systematic review and meta-analysis. J Affect Disord Rep 4:100140. https://doi.org/10.1016/j.jadr.2021. 100140

4. Prigerson HG, Boelen PA, Xu J, Smith KV, Maciejewski PK (2021) Validation of the new DSM-5-TR criteria for prolonged grief disorder and the PG-13-Revised (PG-13-R) scale. World Psychiatry 20:96-106. https://doi.org/10.1002/wps.20823

5. Abbott CH, Prigerson HG, Maciejewski PK (2014) The influence of patients' quality of life at the end of life on bereaved caregivers' suicidal ideation. J Pain Symptom Manage 48:459-464. https:// doi.org/10.1016/j.jpainsymman.2013.09.011

6. Garrido MM, Prigerson HG (2014) The end-of-life experience: modifiable predictors of caregivers' bereavement adjustment. Cancer 120:918-925. https://doi.org/10.1002/cncr.28495

7. Miyajima K, Fujisawa D, Yoshimura K, Ito M, Nakajima S, Shirahase J, Mimura M, Miyashita M (2014) Association between quality of end-of-life care and possible complicated grief among bereaved family members. J Palliat Med 17:1025-1031. https:// doi.org/10.1089/jpm.2013.0552

8. El-Jawahri A, Greer J, Jackson V, Gallagher ER, Kamdar M, Rinaldi S, Temel JS (2019) Depression and anxiety symptoms in bereaved caregivers of patients with advanced cancer. J Clin Oncol 37(31(suppl.)):17. https://doi.org/10.1200/JCO.2019.37. 31_suppl.17

9. Mason TM, Tofthagen CS, Buck HG (2020) Complicated grief: risk factors, protective factors, and interventions. J Soc Work End Life Palliat Care 16:151-174. https://doi.org/10.1080/15524256. 2020.1745726

10. Hales S, Zimmermann C, Rodin G (2008) The quality of dying and death. Arch Intern Med 168:912-918. https://doi.org/10.1001/ archinte.168.9.912
11. Harrop E, Morgan F, Byrne A, Nelson A (2016) "It still haunts me whether we did the right thing": a qualitative analysis of free text survey data on the bereavement experiences and support needs of family caregivers. BMC Palliat Care 15:92. https://doi.org/10. $1177 / 0269216320920533$

12. Holtslander L, Baxter S, Mills K, Bocking S, Dadgostari T, Duggleby W, Duncan V, Hudson P, Ogunkorode A, Peacock S (2017) Honoring the voices of bereaved caregivers: a metasummary of qualitative research. BMC Palliat Care 16:48. https://doi.org/10. 1186/s12904-017-0231-y

13. Curtis JR, Patrick DL, Engelberg RA, Norris K, Asp C, Byock I (2002) A measure of the quality of dying and death: Initial validation using after-death interviews with family members. J Pain Symptom Manage 24:17-31. https://doi.org/10.1016/S08853924(02)00419-0

14. Downey L, Curtis JR, Lafferty WE, Herting JR, Engelberg RA (2010) The Quality of Dying and Death Questionnaire (QODD): empirical domains and theoretical perspectives. J Pain Symptom Manage 39:9-22. https://doi.org/10.1016/j.jpainsymman.2009.05. 012

15. Patrick DL, Engelberg RA, Curtis JR (2001) Evaluating the quality of dying and death. J Pain Symptom Manage 22:717-726. https://doi.org/10.1016/S0885-3924(01)00333-5

16. Francis LE, Kypriotakis G, O'Toole EE, Rose JH (2016) Cancer patient age and family caregiver bereavement outcomes. Support Care Cancer 24:3987-3996. https://doi.org/10.1007/ s00520-016-3219-x

17. Knox MK, Hales S, Nissim R, Jung J, Lo C, Zimmermann C, Rodin G (2017) Lost and stranded: the experience of younger adults with advanced cancer. Support Care Cancer 25:399-407. https://doi.org/10.1007/s00520-016-3415-8

18. Philipp R, Mehnert A, Lo C, Müller V, Reck M, Vehling S (2019) Characterizing death acceptance among patients with cancer. Psychooncology 28:854-862. https://doi.org/10.1002/pon.5030

19. Bovero A, Sedghi NA, Opezzo M, Botto R, Pinto M, Ieraci V, Torta R (2018) Dignity-related existential distress in end-of-life cancer patients: prevalence, underlying factors, and associated coping strategies. Psychooncology 27:2631-2637. https://doi. org/10.1002/pon. 4884

20. Zimmermann C, Swami N, Krzyzanowska M, Hannon B, Leighl N, Oza A, Moore M, Rydall A, Rodin G, Tannock I, Donner A, Lo C (2014) Early palliative care for patients with advanced cancer: a cluster-randomised controlled trial. Lancet 383:1721-1730. https://doi.org/10.1016/s0140-6736(13)62416-2

21. Oken MM, Creech RH, Tormey DC, Horton J, Davis TE, McFadden ET, Carbone PP (1982) Toxicity and response criteria of the Eastern Cooperative Oncology Group. Am J Clin Oncol 5:649_ 655. https://journals.1ww.com/amjclinicaloncology/Abstract/ 1982/12000/Toxicity_and_response_criteria_of_the_Eastern.14. aspx

22. Hales S, Zimmermann C, Rodin G (2010) The quality of dying and death: a systematic review of measures. Palliat Med 24:127144. https://doi.org/10.1177/0269216309351783

23. Heckel M, Bussmann S, Stiel S, Weber M, Ostgathe C (2015) Validation of the German version of the Quality of Dying and Death Questionnaire for informal caregivers (QODD-D-Ang). J Pain Symptom Manage 50:402-413. https://doi.org/10.1016/j. jpainsymman.2015.03.020

24. Faschingbauer T (1981) The Texas Inventory of Grief-Revised. Honeycomb Publishing, Houston

25. Holm M, Alvariza A, Fürst C-J, Öhlen J, Årestedt K (2018) Psychometric evaluation of the Texas Revised Inventory of Grief in a sample of bereaved family caregivers. Res Nurs Health 41:480 488. https://doi.org/10.1002/nur.21886

26. Holm M, Årestedt K, Alvariza A (2019) Associations between predeath and postdeath grief in family caregivers in palliative 
home care. J Palliat Med 22:1530-1535. https://doi.org/10.1089/ jpm.2019.0026

27. Prigerson HG, Horowitz MJ, Jacobs SC, Parkes CM, Aslan M, Goodkin K, Raphael B, Marwit SJ, Wortman C, Neimeyer RA, Bonanno GA, Block SD, Kissane D, Boelen P, Maercker A, Litz BT, Johnson JG, First MB, Maciejewski PK (2009) Prolonged grief disorder: Psychometric validation of criteria proposed for DSM-V and ICD-11. PLoS Med 6:e1000121. https://doi.org/10. 1371/journal.pmed.1000121

28. Prigerson H, Frank E, Kasl S, Reynolds C, Anderson B, Zubenko G, Houck P, George C, Kupfer D (1995) Complicated grief and bereavement-related depression as distinct disorders: preliminary empirical validation in elderly bereaved spouses. Am J Psychiatry 152:22-30. https://doi.org/10.1176/ajp.152.1.22

29. Işıklı S, Keser E, Prigerson HG, Maciejewski PK (2020) Validation of the Prolonged Grief Scale (PG-13) and investigation of the prevalence and risk factors of prolonged grief disorder in Turkish bereaved samples. Death Stud 14:1-11. https://doi.org/10.1080/ 07481187.2020.1745955

30. Pohlkamp L, Kreicbergs U, Prigerson HG, Sveen J (2018) Psychometric properties of the Prolonged Grief Disorder-13 (PG-13) in bereaved Swedish parents. Psychiatry Res 267:560-565. https:// doi.org/10.1016/j.psychres.2018.06.004

31. Tsai W-I, Kuo S-C, Wen F-H, Prigerson HG, Tang ST (2018) Prolonged grief disorder and depression are distinct for caregivers across their first bereavement year. Psychooncology 27:10271034. https://doi.org/10.1002/pon.4629

32. Andresen EM, Malmgren JA, Carter WB, Patrick DL (1994) Screening for depression in well older adults: evaluation of a short form of the CES-D. Am J Prev Med 10:77-84. https://doi.org/10. 1016/S0749-3797(18)30622-6

33. Radloff LS (1977) The CES-D scale: A self-report depression scale for research in the general population. Appl Psychol Meas 1:385-401. https://doi.org/10.1177/014662167700100306

34. Dionne-Odom JN, Azuero A, Lyons KD, Hull JG, Prescott AT, Tosteson T, Frost J, Dragnev KH, Bakitas MA (2016) Family caregiver depressive symptom and grief outcomes from the ENABLE III randomized controlled trial. J Pain Symptom Manage 52:378-385. https://doi.org/10.1016/j.jpainsymman.2016.03.014

35. Kim Y, Carver CS, Spiegel D, Mitchell H-R, Cannady RS (2017) Role of family caregivers' self-perceived preparedness for the death of the cancer patient in long-term adjustment to bereavement. Psychooncology 26:484-492. https://doi.org/10.1002/pon. 4042

36. McDonald J, Swami N, Hannon B, Lo C, Pope A, Oza A, Leighl N, Krzyzanowska MK, Rodin G, Le LW, Zimmermann C (2017)
Impact of early palliative care on caregivers of patients with advanced cancer: cluster randomised trial. Ann Oncol 28:163168. https://doi.org/10.1093/annonc/mdw438

37. Hinton J (1999) The progress of awareness and acceptance of dying assessed in cancer patients and their caring relatives. Palliat Med 13:19-35. https://doi.org/10.1191/026921699672169546

38. Wentlandt K, Burman D, Swami N, Hales S, Rydall A, Rodin G, Lo C, Zimmermann C (2012) Preparation for the end of life in patients with advanced cancer and association with communication with professional caregivers. Psychooncology 21:868-876. https://doi.org/10.1002/pon.1995

39. World Health Organization (2020) Palliative care. https://www. who.int/news-room/fact-sheets/detail/palliative-care. Accessed 10 January 2021.

40. Giesinger JM, Wintner LM, Oberguggenberger AS, Gamper EM, Fiegl M, Denz H, Kemmler G, Zabernigg A, Holzner B (2011) Quality of life trajectory in patients with advanced cancer during the last year of life. J Palliat Med 14:904-912. https://doi.org/10. 1089/jpm.2011.0086

41. Lin H-R, Bauer-Wu SM (2003) Psycho-spiritual well-being in patients with advanced cancer: an integrative review of the literature. J Adv Nurs 44:69-80. https://doi.org/10.1046/j.1365-2648. 2003.02768.x

42. Håkanson C, Öhlén J (2016) Connectedness at the end of life among people admitted to inpatient palliative care. Am J Hosp Palliat Med 33:47-54. https://doi.org/10.1177/1049909114 554077

43. Kastbom L, Milberg A, Karlsson M (2017) A good death from the perspective of palliative cancer patients. Support Care Cancer 25:933-939. https://doi.org/10.1007/s00520-016-3483-9

44. Steinhauser KE, Voils CI, Bosworth H, Tulsky JA (2014) What constitutes quality of family experience at the end of life? Perspectives from family members of patients who died in the hospital. Palliat Support Care 13:945-952. https://doi.org/10.1017/S1478 951514000807

45. Mah K, Shapiro GK, Hales S, Rydall A, Malfitano C, An E, Nissim R, Li M, Zimmermann C, Rodin G (2020) The impact of attachment security on death preparation in advanced cancer: The role of couple communication. Psychooncology 29:833-840. https://doi.org/10.1002/pon.5354

46. Kent EE, Ornstein KA, Dionne-Odom JN (2020) The family caregiving crisis meets an actual pandemic. J Pain Symptom Manage 60:e66-e69. https://doi.org/10.1016/j.jpainsymman.2020.04.006

Publisher's note Springer Nature remains neutral with regard to jurisdictional claims in published maps and institutional affiliations. 